\title{
Analysis of Liquid Medication Dose Errors Made by Patients and Caregivers Using Alternative Measuring Devices
}

\author{
Gyeong Suk Ryu, MS, and Yu Jeung Lee, PharmD, BCPS
}

\begin{abstract}
BACKGROUND: Patients use several types of devices to measure liquid medication. Using a criterion ranging from a $10 \%$ to $40 \%$ variation from a target $5 \mathrm{~mL}$ for a teaspoon dose, previous studies have found that a considerable proportion of patients or caregivers make errors when dosing liquid medication with measuring devices.

OBJECTIVE: To determine the rate and magnitude of liquid medication dose errors that occur with patient/caregiver use of various measuring devices in a community pharmacy.

METHODS: Liquid medication measurements by patients or caregivers were observed in a convenience sample of community pharmacy patrons in Korea during a 2-week period in March 2011. Participants included all patients or caregivers $(\mathrm{N}=300)$ who came to the pharmacy to buy overthe-counter liquid medication or to have a liquid medication prescription filled during the study period. The participants were instructed by an investigator who was also a pharmacist to select their preferred measuring devices from 6 alternatives (etched-calibration dosing cup, printed-calibration dosing cup, dosing spoon, syringe, dispensing bottle, or spoon with a bottle adapter) and measure a $5 \mathrm{~mL}$ dose of Coben (chlorpheniramine maleate/phenylephrine $\mathrm{HCl}$, Daewoo Pharm. Co., Ltd) syrup using the device of their choice. The investigator used an ISOLAB graduated cylinder (Germany, blue grad, $10 \mathrm{~mL}$ ) to measure the amount of syrup dispensed by the study participants. Participant characteristics were recorded including gender, age, education level, and relationship to the person for whom the medication was intended.
\end{abstract}

RESULTS: Of the 300 participants, 257 (85.7\%) were female; 286 (95.3\%) had at least a high school education; and $282(94.0 \%)$ were caregivers (parent or grandparent) for the patient. The mean (SD) measured dose was $4.949(0.378) \mathrm{mL}$ for the 300 participants. In analysis of variance of the 6 measuring devices, the greatest difference from the $5 \mathrm{~mL}$ target was a mean $5.552 \mathrm{~mL}$ for 17 subjects who used the regular (etched) dosing cup and $4.660 \mathrm{~mL}$ for the dosing spoon $(\mathrm{n}=10 ; P<0.001)$. Doses were within $10 \%$ of the $5 \mathrm{~mL}$ target volume for $88.7 \%(n=266)$ of the participant samples. Only 34 cases $(11.3 \%$ ) had dose errors greater than $10 \%$, and only 6 cases $(2.0 \%)$ had a variance of more than $20 \%$ from the $5 \mathrm{~mL}$ target volume. Dose errors greater than $10 \%$ of the target volume were more common for the etched dosing cup $(47.1 \%, n=8)$, the dosing spoon $(50.0 \%$, $n=5)$, and the printed dosing cup $(30.8 \%, n=4)$, but these 3 devices were used by only $13.3 \%$ of the study participants.

CONCLUSIONS: Approximately 1 in 10 participants measured doses of liquid medication with a volume error greater than $10 \%$, and these dose errors were more common with the etched dosing cup, the dosing spoon, and the printed dosing cup. Pharmacists have an opportunity to counsel patients or caregivers regarding the appropriate use of measuring devices for liquid medication.

J Manag Care Pharm. 2012;18(6):439-45

Copyright $\odot 2012$, Academy of Managed Care Pharmacy. All rights reserved.

\section{What is already known about this subject}

- Prescription and over-the-counter medications are often administered for adult and pediatric patients who have difficulty swallowing tablets or capsules.

- Sobhani et al. (2008) found that $67 \%$ of subjects measured an acceptable dose, defined as within 10\% deviation from $5 \mathrm{~mL}$, using an oral syringe versus $15 \%$ using a dosing cup. Simon et al. (1997) found that $67 \%$ of caregivers in a pediatric emergency center measured the amount of acetaminophen accurately (within $\pm 20 \%$ of the intended dose) for their child. Among 302 parents in a public hospital pediatric clinic in New York City, Yin and Mendelsohn, et al. (2010) found a range of accurate dosing (within $20 \%$ of the $5 \mathrm{~mL}$ dose) from $31 \%$ to $94 \%$ for 6 measuring devices; "large" dose errors (greater than 40\%) were made by $26 \%$ of parents using the dosing cup with printed markings and $23 \%$ of parents using the dosing cup with etched markings.

- Medication measuring devices that come in medication containers are regulated by the South Korea Food and Drug Administration, but there are no regulations for measuring devices such as dispensing bottles or oral syringes that pharmacists commonly provide to patients at the time of dispensing.

\section{What this study adds}

- The combined rate of errors in volume greater than $10 \%$ of medication for the 6 measuring devices used by the 300 study participants was $11.3 \%$, but only 6 participants (2.0\%) measured a volume error that was greater than $20 \%$ of the $5 \mathrm{~mL}$ target.

- Dosing spoons, regular dosing cups, and printed dosing cups, all of which are regulated by the South Korea Food and Drug Administration, were associated with relatively high rates of dose errors exceeding $10 \%$ by volume, but these 3 devices were used by only $13 \%$ of the participants for measuring liquid medication.

$\mathrm{O}$ ral medications are commonly prescribed in liquid form to facilitate administration, particularly for adult and pediatric patients who have difficulty swallowing tablets or capsules. Another advantage of liquid medications over tablets or capsules is the enhanced ability to determine individualized dosages. ${ }^{1}$ Various types of liquid medications (e.g., antibiotics, digestive medicine, cold medicine, and fever reducers) are directly sold by pharmacies over the counter (OTC) or by prescription. According to the South Korea 
Food and Drug Administration Notification of the Rules on Medication Safety Containers, Packaging, and Measuring Devices for Drug Administration, the accuracy of calibration for medication measuring devices is required to be within plus or minus $5 \%$ of the indicated value. ${ }^{2}$ The rules are applied only to medication measuring devices that are manufactured in medication containers. According to the regulations, recommended doses in the prescription label directions should be marked on the enclosed medication measuring devices, and a visual test should be conducted for the devices to ensure that the amount of liquid medication measured can be seen clearly.

Yin et al. (2010) evaluated the prevalence of inconsistent dosing directions and measuring devices for pediatric OTC liquid medication and found that $98.6 \%$ of products examined had at least 1 inconsistency, such as superfluous markings and missing markings. ${ }^{3}$ Sobhani et al. (2008) compared the accuracy of an oral syringe with that of a dosing cup for Tylenol (acetaminophen) suspension and found that the mean (standard deviation [SD]) volumes measured with a syringe and cup were $4.5(0.7)$ milliliters $(\mathrm{mL})$ and $6.3(0.7) \mathrm{mL}$, respectively. ${ }^{4}$ Simon and Weinkle (1997) evaluated caregivers' accuracy of OTC liquid medication dosing using acetaminophen in a pediatric emergency center. After conducting a mock dosing scenario with a convenience sampling of 100 caregivers, they found that only $67 \%$ of the caregivers measured the amount of acetaminophen accurately (within $\pm 20 \%$ of the intended dose) for their children. ${ }^{5}$ Yin and Mendelsohn, et al. (2010) assessed parents' liquid medication dosing errors in a public hospital pediatric clinic in New York City and found accurate dosing (within 20\% of the $5 \mathrm{~mL}$ dose) ranged from 31\% to $94 \%$ for 6 measuring devices. ${ }^{6}$ Parents using the dosing cup with printed markings and the dosing cup with etched markings showed lower dosing accuracy rates (31\% and 50\%, respectively) relative to those with other measuring devices (more than 85\%). Liquid medication dosing errors pose a risk to patients from subtherapeutic doses and overdoses. ${ }^{7-9}$

To determine the correct administration of liquid medication, it is necessary to identify the accuracy of patient measurements of liquid medications. Previous studies regarding liquid medication dosing errors did not evaluate the general characteristics of patients or caregivers as factors that could affect the dosing errors. Our purpose was to understand the various factors that might affect liquid medication measurement and to determine the correct administration and dosages by evaluating how the measurements made by patients and caregivers vary according to the medication measuring device used.

\section{Methods}

\section{Study Subjects and Duration}

This study was conducted in a community independent pharmacy in Anyang City, Kyunggido, South Korea, that dispensed

\section{FIGURE 1 Medication Measuring Devices Used in} the Study

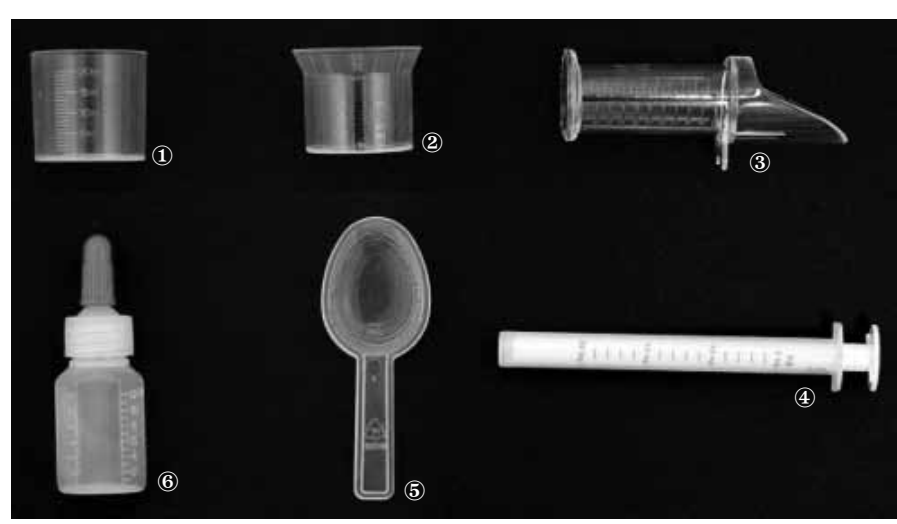

Description:

(1) Etched calibration dosing cup (regular dosing cup): Dosing cup with etched calibration marks for every 1 milliliter $(\mathrm{mL})$ to allow dosing up to $20 \mathrm{~mL}$.

(2) Printed calibration dosing cup: Dosing cup with calibration marks printed in blue with calibration marks of $2.5 \mathrm{~mL}, 3.8 \mathrm{~mL}, 5 \mathrm{~mL}, 7.5$ $\mathrm{mL}$, and $10 \mathrm{~mL}$ in accordance with the dosing instructions for the medication.

(3) Spoon with a bottle adapter: Medication measuring device with a spoon-like end and etched calibration marks for every $0.5 \mathrm{~mL}$. Dosing up to $10 \mathrm{~mL}$ is possible.

(4) Oral syringe: Measures liquid using the same principle as a syringe. The smallest dosing calibration mark is $1.25 \mathrm{~mL}$, and calibration marks are printed for every $0.25 \mathrm{~mL}$. Dosing up to $5 \mathrm{~mL}$ is possible.

(5) Dosing spoon: Calibration marks for every $1 \mathrm{~mL}$ are etched as lines inside the spoon, and a $2.5-\mathrm{mL}$ dosing line is indicated in the middle. Dosing up to $5 \mathrm{~mL}$ is possible.

(6) Dispensing bottle: Calibration marks for every $1 \mathrm{~mL}$ and $2.5 \mathrm{~mL}$ are etched onto both sides, and dosing up to $12 \mathrm{~mL}$ is possible. The cap includes a long cylindrical inlet for the user's convenience during administration.

an average of 300 prescriptions daily. The pharmacy has 2 departments, for prescriptions and OTC medications, and the pharmacy employs 4 full-time pharmacists. The study was a convenience sample of adult patients and caregivers for pediatric patients who visited the pharmacy to purchase OTC medication or receive liquid medication prescribed by a doctor between March 7 and March 20, 2011. In this pharmacy, patients or caregivers who want OTC medications must interact with pharmacists because the purchase counter is located in the prescription section of the pharmacy. We were therefore able to ensure that every patient or caregiver who visited the pharmacy to purchase OTC medication or receive liquid prescription medication when the pharmacist-investigator (Gyeong Suk Ryu) was in the pharmacy was asked to participate in the study. Patients who received single-dose liquid pouches were excluded from the study. 


\begin{tabular}{|c|c|c|c|c|c|c|c|}
\hline \multirow{2}{*}{\multicolumn{2}{|c|}{ Participant Characteristics }} & & \multicolumn{2}{|c|}{ No or Small Dose Errors } & \multicolumn{3}{|c|}{ Large Dose Errors } \\
\hline & & & $<5 \%$ & $6 \%-10 \%$ & $11 \%-20 \%$ & \multicolumn{2}{|c|}{$>20 \%$} \\
\hline \multirow{2}{*}{ Gendera $^{\mathrm{a}}$} & Male & $14.3 \quad(43)$ & $41.9 \quad(18)$ & $32.6 \quad(14)$ & 20.9 & 4.7 & $(2)$ \\
\hline & Female & $85.7 \quad(257)$ & $58.0 \quad(149)$ & $33.1 \quad(85)$ & $7.4 \quad(19)$ & 1.6 & (4) \\
\hline \multirow{6}{*}{$\begin{array}{l}\text { Age }^{\mathrm{b}} \\
\text { (in years) }\end{array}$} & $10-19$ & 0.3 & 100.0 & 0.0 & 0.0 & 0.0 & $(0)$ \\
\hline & $20-29$ & $7.7 \quad(23)$ & $65.2 \quad(15)$ & 34.8 & 0.0 & 0.0 & $(0)$ \\
\hline & $30-39$ & $71.0 \quad(213)$ & $57.7 \quad(123)$ & $33.8 \quad(72)$ & $(15)$ & 1.4 & (3) \\
\hline & $40-49$ & $15.0 \quad(45)$ & $46.7 \quad(21)$ & $35.6 \quad(16)$ & 13.3 & 4.4 & $(2)$ \\
\hline & $50-59$ & 3.0 & 22.2 & 22.2 & 44.4 & 11.1 & (1) \\
\hline & $60-69$ & 3.0 & 55.6 & 11.1 & 33.3 & 0.0 & $(0)$ \\
\hline \multirow{6}{*}{ Relationship to patient ${ }^{c}$} & Father & $9.7 \quad(29)$ & 48.3 & (11) & 13.8 & 0.0 & $(0)$ \\
\hline & Mother & $78.3(235)$ & $59.1 \quad(139)$ & $(79)$ & $(13)$ & 1.7 & $(4)$ \\
\hline & Grandfather & 0.3 & 0.0 & 100.0 & 0.0 & 0.0 & $(0)$ \\
\hline & Grandmother & $5.0 \quad(15)$ & 53.3 & 13.3 & 33.3 & 0.0 & $(0)$ \\
\hline & Self & $6.0 \quad(18)$ & 22.2 & 33.3 & 33.3 & 11.1 & $(2)$ \\
\hline & Other & 0.7 & 100.0 & 0.0 & 0.0 & 0.0 & $(0)$ \\
\hline \multirow{4}{*}{ Educationd } & Elementary school graduate & 0.3 & 0.0 & 0.0 & 100.0 & 0.0 & $(0)$ \\
\hline & Middle school graduate & $4.3 \quad(13)$ & 53.8 & 23.1 & 15.4 & 7.7 & (1) \\
\hline & High school graduate & $29.0 \quad(87)$ & $63.2 \quad(55)$ & $23.0 \quad(20)$ & 10.3 & 3.4 & (3) \\
\hline & College graduate or higher & $66.3(199)$ & $52.8 \quad(105)$ & $38.2 \quad(76)$ & $(16)$ & 1.0 & $(2)$ \\
\hline \multicolumn{8}{|c|}{$\begin{array}{l}\text { aPearson chi-square test for the comparison of numbers of participants in } 4 \text { dose error rate categories according to gender, } P=0.013 \text {. } \\
\text { bPearson chi-square test for the comparison of numbers of participants in } 4 \text { dose error rate categories according to age, } P=0.004 \text {. } \\
\text { cPearson chi-square test for the comparison of numbers of participants in } 4 \text { dose error rate categories according to relationship to patient, } P<0.001 \text {. } \\
\text { dPearson chi-square test for the comparison of numbers of participants in } 4 \text { dose error rate categories according to education, } P=0.015 \text {. }\end{array}$} \\
\hline
\end{tabular}

\section{Research and Evaluation Methods}

This study involved collection of participant characteristics and a liquid medication measurement experiment. Gender, age, education level, and relationship with the patient were reported by the participant, whereas information about the preferred medication measuring devices was acquired using a method in which the study subject made a selection and the researcher entered the choice.

For the liquid medication measurement experiment, the subjects used the preferred medication measuring devices they selected during the survey (an etched-calibration dosing cup [a regular dosing cup], a printed-calibration dosing cup, a dosing spoon, an oral syringe, a spoon with a bottle adapter, or a dispensing bottle) without any recommendations by the pharmacist. The participants were asked by the pharmacistinvestigator to measure $5 \mathrm{~mL}$ of liquid medication with routine instructions, and their measurements were verified by the pharmacist-investigator (Gyeong Suk Ryu) measuring the same sample with a graduated cylinder. Of the medication measuring devices used in the experiment, 4 types (the regular dosing cup, the printed dosing cup, the dosing spoon, and the oral syringe) were packaged with the medicines in accordance with South Korea Food and Drug Administration provisions, whereas the dispensing bottle and the spoon with the bottle adapter were purchased separately by the pharmacy and provided to the patients free of charge (Figure 1).
For the liquid medication measurement experiment, we used Coben (chlorpheniramine maleate/phenylephrine $\mathrm{HCl}$, Daewoo Pharmaceutical Co., Ltd, Busan, South Korea) syrup, which is the liquid medication that is most frequently prescribed and prepared at the pharmacy. It is available OTC as well as by prescription in South Korea. An ISOLAB graduated cylinder (Germany, blue grad, $10 \mathrm{~mL}$ ) was used to measure the amount of syrup dispensed by the study participants. One researcher (Gyeong Suk Ryu) read the measured doses using the graduated cylinder.

Liquid medication dosing error rates were classified as less than $5 \%, 6 \%$ to $10 \%, 11 \%$ to $20 \%$, and more than $20 \%$ (the base measured dose of $5 \mathrm{~mL}$ was the target). We defined volume errors of $10 \%$ or less as small and volume errors greater than $10 \%$ as large. The U.S. Pharmacopeia specifies that the volume error incurred in measuring $5 \mathrm{~mL}$ of liquid medication with calibrated devices "should be not greater than $10 \%$ of the indicated amount,"10 and Sobhani et al. defined "acceptable dose" as within $10 \%$ of the indicated amount. ${ }^{4}$

SPSS 18.0 (IBM SPSS, Armonk, NY) was used to perform a frequency analysis of demographic characteristics (gender, age, education level, and relationship with the patient) and the medication measuring device preferences, and the mean and $\mathrm{SD}$ were determined for the measured dose of the liquid. The Pearson chi-square test was performed for the differences in dosing error rates according to the participant characteristics, 


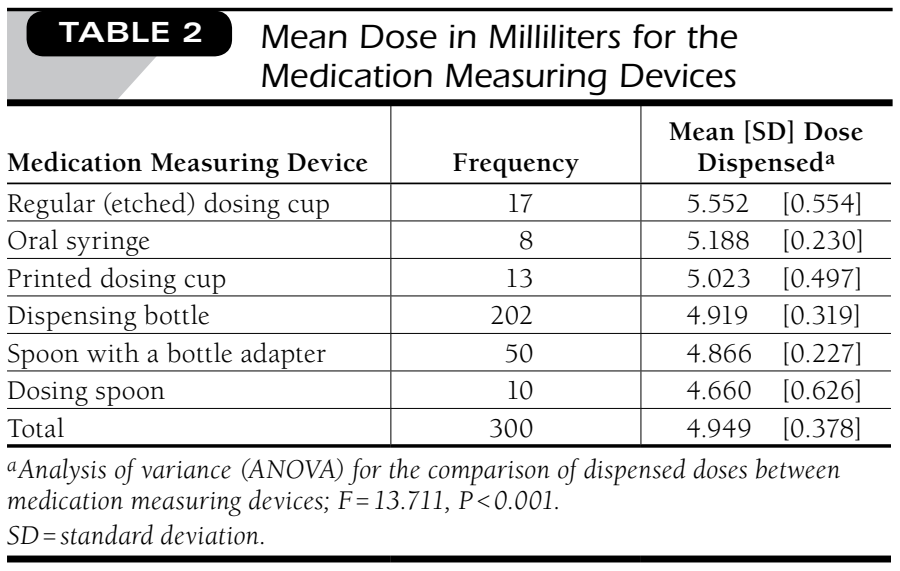

and analysis of variance (ANOVA) or an independent $t$ test was performed for the differences in measured liquid medication doses according to the participant characteristics. In addition, a frequency analysis was performed for dosing error rates according to the preferred medication measuring device.

\section{Results}

\section{General Characteristics of the Respondents}

Of the 347 people asked by the pharmacist-investigator, 300 patients and caregivers agreed to participate in the study. The majority of the participants were female $(85.7 \%, \mathrm{n}=257)$, and $97.0 \%(n=291)$ were younger than age 60 (Table 1). In addition, most of the participants were the mothers of the patients for whom the medicine was intended $(78.3 \%, \mathrm{n}=235)$, and only $18(6.0 \%)$ of the participants were the patients themselves. All but 14 participants $(4.7 \%)$ reported education level of at least high school.

\section{Analysis of the Measured Dose by Device}

The mean (SD) measured dose was $4.949(0.378) \mathrm{mL}$ for the 300 study subjects who were instructed to measure $5 \mathrm{~mL}$ of liquid medication (Table 2). The mean (SD) measured dose for males was $5.119(0.505) \mathrm{mL}$ versus $4.920(0.346) \mathrm{mL}$ for females $(P<0.001$, data not presented in Table 2$)$. The greatest difference from the $5 \mathrm{~mL}$ target was observed for the regular dosing cup, which had a mean measured dose of $5.552 \mathrm{~mL}$. The dosing spoon showed the next highest difference from the target $(-0.340 \mathrm{~mL})$, and the printed dosing cup and dispensing bottle showed the least differences from the $5 \mathrm{~mL}$ target, $+0.023 \mathrm{~mL}$ and $-0.081 \mathrm{~mL}$, respectively (Table 2 ).

\section{Description of Dosing Errors by Participant Characteristics and Device}

Two hundred sixty-six (88.7\%) samples were within $10 \%$ of the $5 \mathrm{~mL}$ target volume, defined as no or small errors. Large errors greater than $10 \%$ from the target volume numbered 34 (11.3\%, Table 3). There were statistically significant differences in dose error rates based on gender, age, education level, or relationship to the patient (Table 1). Large dose errors greater than $10 \%$ were more common for males $(25.6 \%, \mathrm{n}=11)$, patients aged $50-59$ years $(55.5 \%, n=5)$, patient-self $(44.4 \%, n=8)$, and elementary school education level $(100.0 \%, \mathrm{n}=1)$. However, the distribution of the study participants in each category of participant characteristic was quite variable.

Three devices had a high proportion of large volume errors: the dosing spoon $(50 \%, n=5)$, the regular (etched) dosing cup $(47.1 \%, n=8)$, and the printed dosing cup $(30.8 \%, n=4$; Table 3). Volume errors greater than $20 \%$ of the $5 \mathrm{~mL}$ target occurred among only 6 participants (2.0\% collectively for 3 devices), and none of these large dose errors occurred in the oldest age group (aged 60-69).

\section{Discussion}

In this study, the percentage of adults (patients themselves) was low because the majority of their prescribed liquid medications were single-dosage pouches; therefore, they were ineligible for participation, and the pharmacy primarily handled pediatric prescriptions. Most of the pediatric patient caregivers were in the age category of 30-39 years, and 95\% of the participants had at least a high school education.

The mean measured dose for 8 (44\%) of the adult patients themselves deviated from the target $5 \mathrm{~mL}$ amount by more than $10 \%$, and 2 of these patients accounted for one-third of the total of 6 patients who had an error in dose-volume greater than $20 \%$. This may have occurred because patients do not measure their own doses as carefully as parents and caregivers measure doses for children. ${ }^{11,12}$

Compared with other studies of liquid medication dosing, our findings show relatively little variance from the $5 \mathrm{~mL}$ target volume. In our study, 266 participants (88.7\%) measured a volume of liquid that was within $10 \%$ of the target, and 294 participants (98.0\%) measured a volume that was within 20\% of the target. Sobhani et al. found that $66.7 \%$ of subjects using the oral syringe and $14.6 \%$ of subjects using the dosing cup measured an acceptable dose, defined as no more than 10\% deviation from $5 \mathrm{~mL} .{ }^{4}$ Simon et al. found that $67 \%$ of caregivers in a pediatric emergency center measured the amount of acetaminophen accurately (within $\pm 20 \%$ of the intended dose) for their children. ${ }^{5}$ Among 302 parents in a public hospital pediatric clinic in New York City, Yin and Mendelsohn, et al. found that a range from $31 \%$ to $94 \%$ measured an accurate dose (within 20\% of the $5 \mathrm{~mL}$ dose) for 6 measuring devices; "large" dosing errors (greater than $40 \%$ ) were made by $26 \%$ of parents using the dosing cup with printed markings and 23\% of parents using the dosing cup with etched markings. ${ }^{6}$

Sobhani et al., comparing the accuracy of an oral syringe with a dosing cup, found that $66.7 \%$ of participants measured a dose within $10 \%$ of the indicated dose with the oral syringe versus $14.6 \%$ with the dosing cup. ${ }^{4}$ Our results were similar, 
Analysis of Liquid Medication Dose Errors Made by Patients and Caregivers Using Alternative Measuring Devices

TABLE 3 Dose Error Rates by Medication Measuring Device

\begin{tabular}{|c|c|c|c|c|c|c|c|}
\hline \multirow{3}{*}{$\begin{array}{l}\text { Medication Measuring Device } \\
\text { Regular (etched) dosing cup }(n=17)\end{array}$} & \multirow{3}{*}{$\frac{\text { Range (in mL) }}{4.6-6.4}$} & \multicolumn{2}{|c|}{ No or Small Dose Errors } & \multicolumn{4}{|c|}{ Large Dose Errors } \\
\hline & & $<5 \%$ & $6 \%-10 \%$ & \multicolumn{2}{|c|}{$11 \%-20 \%$} & \multicolumn{2}{|c|}{$>20 \%$} \\
\hline & & 29.4 & 23.5 & 23.5 & (4) & 23.5 & (4) \\
\hline Dosing spoon $(\mathrm{n}=10)$ & $3.7-5.6$ & 30.0 & 20.0 & 40.0 & (4) & 10.0 & (1) \\
\hline Printed dosing cup $(\mathrm{n}=13)$ & $4.4-5.9$ & 15.4 & 53.8 & 30.8 & (4) & 0.0 & $(0)$ \\
\hline Dispensing bottle $(\mathrm{n}=202)$ & 3.8-5.7 & $59.9 \quad(121)$ & $32.7 \quad(66)$ & 6.9 & (14) & 0.5 & (1) \\
\hline Spoon with a bottle adapter $(\mathrm{n}=50)$ & $4.5-5.6$ & 64.0 & $32.0 \quad(16)$ & 4.0 & (2) & 0.0 & $(0)$ \\
\hline Oral syringe $(n=8)$ & 4.8-5.4 & 50.0 & 50.0 & 0.0 & $(0)$ & 0.0 & $(0)$ \\
\hline All devices $(\mathrm{N}=300)$ & $3.7-6.4$ & $55.7 \quad(167)$ & $33.0 \quad(99)$ & 9.3 & $(28)$ & 2.0 & (6) \\
\hline
\end{tabular}

with no measured volumes outside of $10 \%$ of the $5 \mathrm{~mL}$ target for the oral syringe versus $47 \%$ for the regular (etched) dosing cup, but the number of cases for both devices was small in our study ( $\mathrm{n}=8$ for each).

In our study, we found high rates of inaccurate dosing with the dosing spoon, the regular dosing cup, and the printed dosing cup, which may have resulted from difficulty in seeing the calibration markings while measuring. Variance in measured doses was low for the spoon with bottle adapter $(S D=0.227)$, oral syringe ( $\mathrm{SD}=0.2300)$, and dispensing bottle $(\mathrm{SD}=0.319)$. Participants noted inconvenience using the oral syringe.

The definition of error in liquid medication dosing varies considerably among studies. Yin and Mendelsohn, et al. defined "accurate dosing" as within 20\% of the target $5 \mathrm{~mL}$ volume, a "small error" as 20\%-40\% variance from the $5 \mathrm{~mL}$ target, and more than $40 \%$ from the target volume as a "large error." In our study, the mean (SD) measured dose with use of a regular dosing cup was $5.552(0.554) \mathrm{mL}$, compared with 7.0 (3.2) $\mathrm{mL}$ in Yin and Mendelsohn, et al. Actual measured volumes not within $20 \%$ of the target were made by $49.8 \%$ of participants $(n=150)$ using a dosing cup with etched calibration markings in the study by Yin and Mendelsohn, et al., compared with 4 of 17 participants (23.5\%) who had dosing errors greater than $20 \%$ with use of the dosing cup in our study. Dose errors greater than $20 \%$ for the dosing spoon and the oral syringe also occurred less frequently in our study $(10.0 \%$ and $0.0 \%$, respectively) than in Yin and Mendelsohn, et al. (14.0\% and $8.6 \%$, respectively ${ }^{6}$.

Frush et al. (2004) found that the mean deviation from the recommended dose range for OTC acetaminophen liquid was lower in the caregiver group given color coding of dose zones compared with conventional dosing methods (0.5\% vs. $29 \%$, respectively). ${ }^{13}$ Peacock et al. (2010) found that fewer patients made measurement errors after pharmacist instruction on the proper use of measurement devices; measurement error was less common with a pharmacist-supplied dose syringe compared with the manufacturer-supplied device; and the measurement error rate was higher for nystatin suspension than for the less-viscous digoxin elixir. ${ }^{14}$ With an error defined as at least 20\% deviation from the intended dose, errors in dose before and after pharmacist instruction were made by $88.0 \%$ and $85.6 \%$ of patients for nystatin, respectively, and by $24.8 \%$ and $4.0 \%$ of patients for digoxin elixir, respectively. ${ }^{14}$

When pharmacists administer liquid medications at pharmacies in South Korea, it is common practice to attach a label or write information on the container about the drug name and its purpose, dosage, and frequency. In addition, pharmacists in South Korea provide medication guidance and make efforts to reduce liquid medication dosing errors by highlighting important information (e.g., keeping the liquid refrigerated and dosage differences from other medications) with a colored pen, indicating the recommended dosage on the calibration markings on the medication measuring devices for older patients and dividing and packaging the prescription into individual doses for patients and caregivers who have difficulties with measurements. Pharmacists, however, do not know if patients get enough information on how to measure liquid medications accurately. There has been no study conducted in South Korea in terms of liquid medication dosing, but many patients either do not receive a sufficient explanation of how to read the calibration markings on the medication measuring devices, or their use of the information on the medicine containers is insufficient based on studies conducted in other countries. ${ }^{5,15,16}$ During the course of this study, we observed the study participants measuring liquid medication by placing the medication measuring devices on the counter and looking at the calibration markings from above or below rather than measuring the liquid with the calibration markings at eye level.

Of the 6 types of medication measuring devices used in the present study, the dosing spoon, regular dosing cup, and printed dosing cup are regulated by the South Korea Food and Drug Administration and had higher rates of dosing errors than the other measuring devices, but there were only 40 participants who used these 3 measuring devices. These 3 devices were selected less often compared with the spoon with bottle adapter $(n=50)$ and the dispensing bottle $(n=202)$.

Liquid dosing errors can be clinically significant, particularly for infants and the elderly. Underdosing can result in lack 
of therapeutic response, and overdosing can result in adverse drug events. ${ }^{7}$ Medications with a narrow therapeutic index such as phenytoin or digoxin require particular attention to accurate dosing. . $^{17-21}$

\section{Limitations}

There are several threats to the validity of our findings. Foremost among the limitations is the convenience sample and the bias toward selection of adults with an education level that was on average much higher than that of the adult population in South Korea. In the present study sample, about 5\% of participants had an elementary or middle-school education, compared with $20 \%$ in South Korea as a whole, and two-thirds had a college or higher-level education, compared with 39\% in South Korea as a whole. ${ }^{22}$ The percentage of participants in the Yin and Mendelsohn, et al. study who had an educational level beyond high school was $51.0 \%$, compared with $95.3 \%$ in our study. This difference is large and could have affected the results.

Second, there was nonrandom selection of measuring devices by study participants and a small sample size (less than 20) for each of 4 of the measuring devices. Third, there is the possibility that the participants measured the dose correctly based on the device used, but the device may have been calibrated incorrectly. Fourth, asking the subjects to measure the liquid in the presence of the pharmacist-investigator may have affected the dose error rates. Fifth, we may have underestimated the rate of dose errors because of the small number of older participants in the study. More studies of accuracy in liquid medication dosing may help identify risk factors for dose errors to help pharmacists target at-risk patients and caregivers.

Sixth, our results may have also been affected by the relatively low viscosity of the syrup used in this study and may not be representative of higher viscosity liquid medications. Finally, the generalizability of our findings is limited by the conduct of this study in 1 pharmacy located in a metropolitan area in South Korea.

\section{Conclusions}

The present study found that the dosing error rate was $11.3 \%$ for volume deviation greater than $10 \%$, suggesting that most patients and caregivers accurately measured liquid medications. However, we observed differences in dosing error rates among the various medication measuring devices, with major (greater than $10 \%$ variance) volume errors occurring more often with dosing spoons (50\%) or regular dosing cups (47\%) compared with printed dosing cups (31\%), dispensing bottles $(7 \%)$, spoons with a bottle adapter (4\%), and oral syringes ( $0 \%)$. There is an opportunity for community pharmacists to educate patients and caregivers on the accurate use of medication measuring devices.

\section{Authors}

GYEONG SUK RYU, MS, is Researcher, Sookmyung Women's University, Seoul, South Korea; and YU JEUNG LEE, PharmD, BCPS, is Assistant Professor, College of Pharmacy, Kangwon National University, Gangwon-do, South Korea.

AUTHOR CORRESPONDENCE: Yu Jeung Lee, PharmD, BCPS, College of Pharmacy, Kangwon National University, Kangwondaehak-gil 1, Chuncheon-si, Gangwon-do 200-701, South Korea.Tel.: 82.33.250.6910; E-mail:yujeunglee@yahoo.com.

\section{DISCLOSURES}

This research was performed without external funding. Lee designed the study with the assistance of Ryu, and Ryu collected the data as the onsite pharmacist-investigator. Lee and Ryu interpreted the data and wrote and revised the manuscript.

\section{REFERENCES}

1. Hurst M. Oral medication dispensers in clinical research. J Clin Res Best Pract. 2006;2(9):1-3. Available at: http://staging.baxa.com/resources/ docs/0609_Oral_Dispenser.pdf. Accessed June 15, 2012

2. South Korea Food and Drug Administration. Rules on medication safety containers, packaging and measuring devices for drug administration, Notification No. 2011-4, 2011.2.1 [in Korean]. Available at: http://node24 www-kfda-go-kr.nciashield.org/index.kfda? $\mathrm{cmd}=\mathrm{v} \& \mathrm{mid}=92 \&$ pageNo=6\&s eq=3424. Accessed June 15, 2012.

3. Yin HS, Wolf MS, Dreyer BP, et al. Evaluation of consistency in dosing directions and measuring devices for pediatric nonprescription liquid medications. JAMA. 2010;304(23):2595-602. Available at: http://jama.jamanetwork.com/article.aspx?doi=10.1001/jama.2010.1797. Accessed June 15, 2012.

4. Sobhani P, Christopherson J, Ambrose PJ, Corelli RL. Accuracy of oral liquid measuring devices: comparison of dosing cup and oral dosing syringe. Ann Pharmacother. 2008;42(1):46-52

5. Simon HK, Weinkle DA. Over-the-counter medications: do parents give what they intend to give? Arch Pediatr Adolesc Med. 1997;151(7):654-56.

6. Yin HS, Mendelsohn AL, Wolf MS, et al. Parents' medication administration errors: role of dosing instruments and health literacy. Arch Pediatr Adolesc Med. 2010;164(2):181-86. Available at: http://archpedi.jamanetwork.com/ article.aspx?doi=10.1001/archpediatrics.2009.269. Accessed June 15, 2012.

7. Madlon-Kay DJ, Mosch FS. Liquid medication dosing errors. J Fam Pract. 2000;49(8):741-44. Available at: http://www.jfponline.com/Pages. asp?AID=2582. Accessed June 15, 2012.

8. Eliopoulos GM, Moellering RC Jr. Principles of antibiotic therapy. Med Clin North Am. 1982;66(1):3-15.

9. Stamey TA, Bragonje J. Resistance to nalidixic acid. A misconception due to underdosage. JAMA. 1976;236(16):1857-60.

10. US Pharmacopeia. <1221> Teaspoon (definition). Available at: http://www. pharmacopeia.cn/v29240/usp29nf24s0_cl221.html. Accessed June 15, 2012.

11. Zandieh SO, Goldmann DA, Keohane CA, Yoon C, Bates DW, Kaushal R. Risk factors in preventable adverse drug events in pediatric outpatients. $J$ Pediatr. 2008;152(2):225-31.

12. Schaefer MK, Shehab N, Cohen AL, Budnitz DS. Adverse events from cough and cold medications in children. Pediatrics. 2008;121(4):783-87.

13. Frush KS, Luo X, Hutchinson P, Higgins JN. Evaluation of a method to reduce over-the-counter medication dosing error. Arch Pediatr Adolesc Med. 2004;158(7):620-24. Available at: http://archpedi.jamanetwork.com/article. aspx?doi=10.1001/archpedi.158.7.620. Accessed June 15, 2012. 
14. Peacock G, Parnapy S, Raynor S, Wetmore S. Accuracy and precision of manufacturer-supplied liquid medication administration devices before and after patient education. J Am Pharm Assoc (2003). 2010;50(1):84-86.

15. Gribetz B, Cronley SA. Underdosing of acetaminophen by parents. Pediatrics. 1987;80(5):630-33.

16. McMahon SR, Rimsza ME, Bay RC. Parents can dose liquid medication accurately. Pediatrics. 1997;100(3 Pt 1):330-33.

17. Hvidberg EF, Dam M. Clinical pharmacokinetics of anticonvulsants. Clin Pharmacokinet. 1976;1(3):161-88.

18. Battino D, Estienne M, Avanzini G. Clinical pharmacokinetics of antiepileptic drugs in paediatric patients. Part II. Phenytoin, carbamazepine, sulthiame, lamotrigine, vigabtrin, oxcarbazepine and felbamate. Clin Pharmacokinet. 1995;29(5):341-69.
19. Lund L, Alvan G, Berlin A, Alexanderson B. Pharmacokinetics of single and multiple doses of phenytoin in man. Eur J Clin Pharmacol. 1974;7(2):81-86. 20. Smith TW. Digitalis toxicity: epidemiology and clinical use of serum concentration measurements. Am J Med. 1975;58(4):470-76.

21. Smith TW, Haber E. Digoxin intoxication: the relationship of clinical presentation to serum digoxin concentration. J Clin Invest. 1970;49(12):2377-86.

22. Organization for Economic Co-operation and Development. Education at a Glance 2011: OECD Indicators. OECD Publishing; 2011. Available at: http:// dx.doi.org/10.1787/eag-2011-en. Accessed June 15, 2012. 